\title{
Imaging Buried Monolayers at Atomic Resolution using Electron Channeling
}

L. Fitting, ${ }^{*}$ M. Jones, ${ }^{*}$ V. Vaithyanathan, ${ }^{* *}$ D. G. Schlom, ${ }^{* *}$ and D. A. Muller*

* Applied and Engineering Physics, Cornell University

** Department of Material Science, Pennsylvania State University

Electron channeling in a crystal can in principle enhance the probe intensity at the exit surface $[1,2]$, thus leading to increased visibility of adatoms [2] or dopant atoms [3]. Here we use this effect to tune the contrast of buried monolayers by selecting the thickness of the crystal substrate to be close to the channeling maximum where entrance-surface effects are suppressed and the visibility of the buried monolayers is enhanced. Our theoretical and experimental studies provide us with new tools for studying nucleation and the early stages of crystal growth, which we apply to the formation of thin oxide crystals on Si.

We have conducted multislice simulations to study the effect of beam focusing in $\operatorname{Si}(100)$ crystals. Fig. 1 shows the beam profile at the entrance and at the exit surface of a $15 \mathrm{~nm}$ thick, [100] zone-axis Si crystal. As the probe propagates through the crystal, a sharp channeling peak forms and the FWHM decreases by a factor of three to $\sim 0.4 \AA$. Here, the thickness of the crystal was chosen, so that it coincides with the channeling maximum (Fig. 2). Due to the large intensity of the channeling peak, an atom placed on a Si column at the exit surface of the Si crystal should contribute significantly more ( $\sim 4$ times) to the annular dark field (ADF) signal than at the entrance surface. Indeed, the visibility of a single Sr atom on a $15 \mathrm{~nm}$ thick Si crystal simulated by multislice is strongly enhanced if the probe propagates through the crystal first before scattering off of the $\mathrm{Sr}$ atom. However, if the $\mathrm{Sr}$ atom is misplaced from the atomic column its visibility quickly decreases, tracking the channeling probe profile (Fig. 3).

To explore this effect experimentally, we grew 2.5uc $\mathrm{SrTiO}_{3}$ on $\mathrm{Si}(100)$, capped with $15 \mathrm{~nm}$ a-Si using MBE. While these layers have been studied during growth, a serious question for such thin layers is if their structure remains unaltered after they have been overgrown. Fig. 4 shows plan view ADF-STEM images of the thin, buried $\mathrm{SrTiO}_{3}$ film where the sample was oriented so that the crystalline substrate constituted the entrance surface. We find the formation of islands of the $\mathrm{SrTiO}_{3}$ film after capping with a-Si. Fig. $4 \mathrm{~b}$ shows perovskite lattice fringes in the bright region confirming that these patches are due to $\mathrm{SrTiO}_{3}$. The contrast of the $\mathrm{SrTiO}_{3}$ islands can be maximized by choosing a region of the sample where the thickness of the crystalline $\mathrm{Si}$ is about $15 \mathrm{~nm}$ which corresponds to maximum channeling. The average island thickness of 4uc (Fig. 4c) is consistent with a non-uniform $\mathrm{SrTiO}_{3}$ coverage. When the sample was turned up-side-down so that the beam passed through the a-Si first, we were not able to resolve the $\mathrm{SrTiO}_{3}$ lattice. In this geometry, narrowing of the probe due to channeling will occur only after the beam has passed through the $\mathrm{SrTiO}_{3}$ layer and will not lead to enhanced visibility of this layer.[4]

\section{References}

[1] J. Fertig, H. Rose, Optik 59 (1981) 407.

[2] R. Loane, E. J. Kirkland, J. Silcox, Acta Cryst. A44 (1988) 912.

[3] P. M. Voyles, D. A. Muller, E. J. Kirkland, Microsc. Microanal. 10 (2004) 291.

[4] We acknowledge support under the ONR EMMA MURI monitored by Colin Wood, by the Cornell Center for Materials Research (IRG-3) and by Applied Materials (LF). 


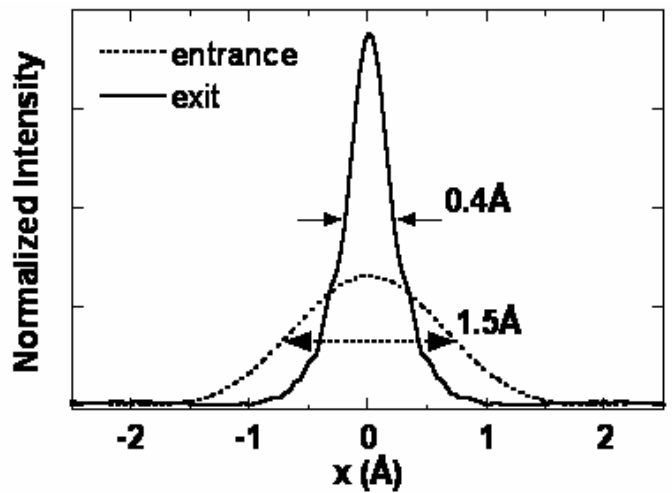

Fig. 1. Sharpening of an electron probe due to channeling in a crystal. Probe profile at the entrance and at the exit surface of a $15 \mathrm{~nm}$ thick, [100] zone-oriented Si crystal, respectively.

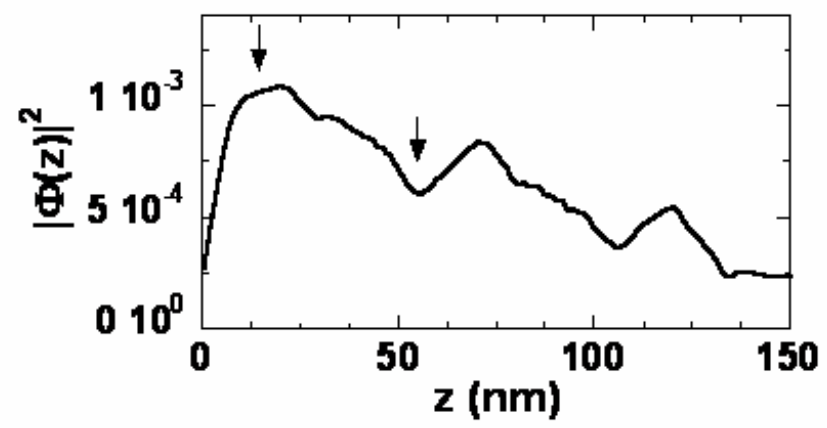

Fig. 2. Channeling pattern for Si [100], where the probe intensity $|\Phi(z)|^{2}$ at thickness $\mathrm{z}$ is proportional to the change of the ADF intensity with respect to $\mathrm{z}$.

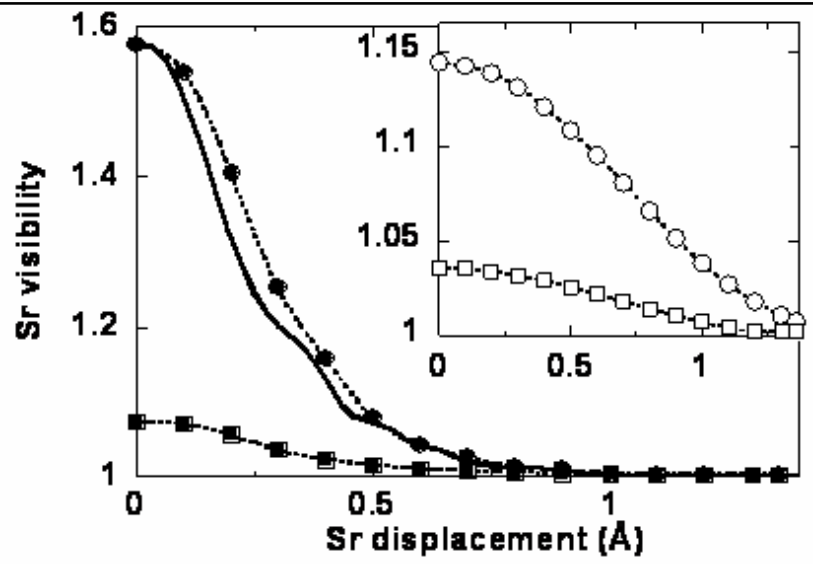

Fig. 3. Multislice simulations of the visibility of a single $\mathrm{Sr}$ atom misplaced for an atomic column of a $15 \mathrm{~nm}$ (dots) and $55 \mathrm{~nm}$ (squares) Si crystal, respectively. The visibility decreases as the probe intensity (solid line) at the exit surface. The inset shows the calculated visibilities according to the incoherent imaging model.
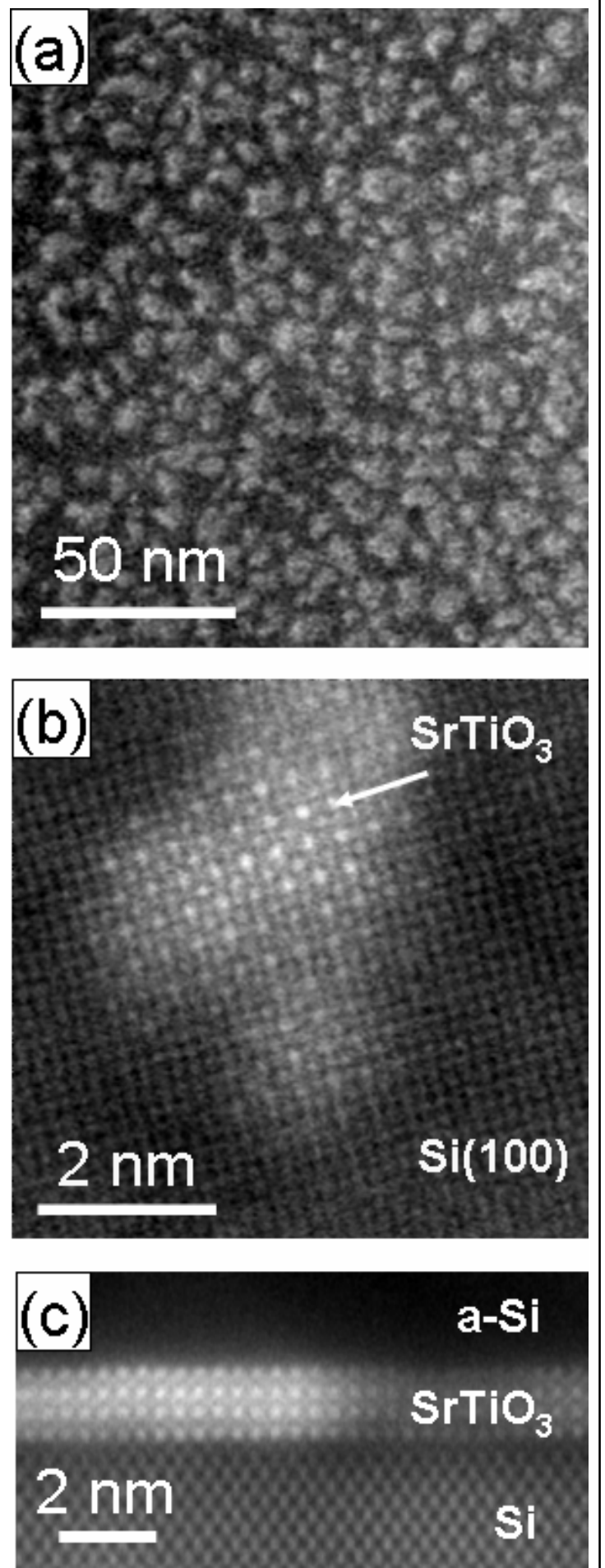

Fig. 4. ADF-STEM image of 2.5uc SrTiO3 grown on $\mathrm{Si}(100)$ and capped with $15 \mathrm{~nm}$ a-Si. (a), (b) Plan view STEM shows the formation of $\mathrm{SrTiO}_{3}$ islands and a non-uniform coverage. (c) The average thickness of the islands is 4uc as found by cross sectional STEM. 